\title{
The Development and Current Condition of TCM Orthopedic Informatization in China
}

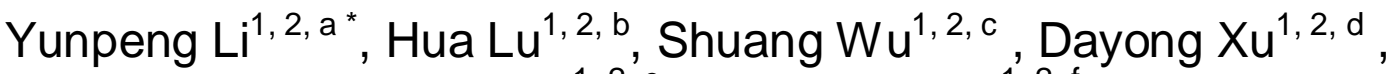 \\ Zongjin Yue ${ }^{1,2, e}$ and Xinli Wang ${ }^{1,2, f}$
}

${ }^{1}$ Henan Province Hospital of Traditional Chinese Medicine, 450002.Zhengzhou, 450000, Henan, P. R. China

${ }^{2}$ The Second Affiliated Hospital of Henan University of Traditional Chinese Medicine, 450000, Henan, P. R. China

\author{
a253863528@qq.com, luhuahntcm@163.com, \\ cwushuanghntcm@163.com, ,xudayonghntcm@163.com \\ eyuezongjinhntcm@163.com ,'wangxinli@163.com
}

Keywords: TCM; Orthopedic; Informatization; China; Condition

\begin{abstract}
With the gradual development of medical technology in China, the computer is widely used in daily medical treatment. The most extensive information management has now formed a more standardized information management system, which includes medical technology information, care level information and so on. At present, China's traditional Chinese medicine orthopedic information management system platform has brought a new pattern of Chinese medicine orthopedic information construction, and promoted the comprehensive improvement of TCM orthopedic management level. Today's society is information society, and it is necessary to serve as the hospital and the simultaneous development of information society into the era of information technology orthopedics.
\end{abstract}

\section{Information}

Is the most important scientific and technological resources of medical work, the degree of information to determine the degree of development of departments, fully functional information through the relevant departments of information collection, collation, analysis and application; you can always grasp the forefront of professional technology and the needs of patients ${ }^{[1]}$. Improve the level of medical technology, expand the medical market, in the industry to create first-class competition in technology, first-class equipment, first-class service, first-class environment, first-class management, first-class benefits, boost the orthopedic construction has always maintained rapid scientific development Construction is of great significance to enhance the quality of medical and health services, to facilitate people to seek medical treatment, innovative service model, and to strengthen service supervision ${ }^{[2]}$.

\section{On the Connotation of Informative Orthopedic Construction}

Information from the 1967 government of Japan, a research agency information, October 24, 2002 China's national information leading group approved the promulgation of the "National Economic and Social Development Tenth Five-Year Plan Information Focus on special planning ${ }^{[3]}$. At present, the information on orthopedics has not yet a clear definition, I personally understand the realization of information technology orthopedics at least include the following three aspects: (1) "6S" technology in the department has been widely used and leading, that is, digital imaging diagnosis (DRIS), Digital Navigation System (DNS), Digital Endoscopy System (DES), Computer Assisted Orthopedic Surgery System (CAOS), Orthopedic Medical Workstation Information System (HMIS) for Hospital Management Information System Terminal, Remote Network System RNS) (referred to as 6S), support from the modern orthopedic three-dimensional multi-dimensional information field, the traditional medical model has undergone a qualitative change; (2) the basic form of the 
information network as the basis for information support for information, talent, (3) the establishment of a full range of cultural and related communications and film and television technology facilities, including department rooms and subscriptions of books, magazines, newspapers (paper version / electronic (electronic), the quality of the system, Version) and their own website, e-mail, telex, multimedia, photography, video, recording, scanning and other commonly used information equipment, Should also be equipped with a person responsible. The limitations of the various aspects of the medical work are gradually disappearing, and the communication between the medical, educational, research, medical, technical and medical personnel is promoted, which greatly improves the work efficiency, the quality of work and the effect. At present, the top three hospital orthopedic hospital information management system and the Department of information technology hardware and software configuration in general

\section{The Development of Orthopedic Telemedicine in China}

China's orthopedic telemedicine activities began in the 1990s. At the end of 1995, the Beijing Municipal Defense Science and Technology Commission 514 hospital using satellite systems and the United States opened trans-Pacific spine surgery conducted a remote case discussion. In March 1996, Shandong girl Yang Xiaoxia because of unruffled arm to the Beijing Military Region General Hospital orthopedic medicine, consultation doctors encountered difficulties, through the Internet to the international community for help, and soon more than 200 pieces of information from all over the world spread to Beijing, the cause of the final Was diagnosed as a muscle bacteria, effectively shorten the course of the disease. The above-mentioned remote consultation in the country caused great repercussions and more Chinese orthopedic doctors from the Internet and telemedicine ${ }^{[4]}$. At present, most of China's top three hospitals orthopedic can be completed through the network remote, real-time dynamic TV live medical consultation work. March 5, 2006, Beijing Jishuitan Hospital Orthopedics through the network remote command, by the Yan'an University Hospital doctors on the ground operation, a severe traumatic hip dislocation, left tibial and fibular fractures, pelvic fractures in patients with remote surgery, the successful completion of Treatment of fracture and interlocking intramedullary nail fixation. In recent years due to sudden natural disasters, such as Wenchuan earthquake, Yushu earthquake, a variety of major industrial and mining accidents, traffic accidents caused by a large number of orthopedic wounded, through a variety of modern information systems in the first time to achieve a remote orthopedic consultation Or dispatch experts to the scene to participate in emergency treatment, greatly improving the success rate of treatment and treatment. According to media reports, by the Third Military Medical University Xinqiao Hospital and the Institute of Shenyang Institute of Automation jointly developed with independent intellectual property rights of the spine minimally invasive surgical robot, has been in July 11, 2010 into the early clinical trials, this type of robot also Will be fully automated, remote automation and intelligent development, in the battlefield, disaster relief and other harsh environments to achieve remote operation remote control.

\section{The Development and Status Quo of Orthopedic Distance Education in China}

Medicine is a need for continuous learning, deepening of science, especially specialists, continues to learn and grow the environment is very important. China's talent training methods, mainly "in the post" to learn, due to study abroad opportunities, time, cost and other resource factors, a direct impact on the growth rate of a large number of young doctors, through the remote network information platform, Education, not only low cost, sustainable, and massive information, to speed up the growth and development of specialist. However, in the course of the development of orthopedic distance education, insufficient funds and lack of relevant teachers are the main problems that plague the development of distance education ${ }^{[5]}$. At present, only a small number of teaching hospitals orthopedic practices of remote real-time teaching mode. In ensuring the quality of distance education, to be further explored and improved. In addition, online education is a two-way interactive teaching process, the information literacy of online educators is very important, 
such as the ability to use information tools, the ability to obtain information, the ability to handle information, generate information, create information, Information ability, information collaboration awareness and ability and information immunity. In the process of network teaching, only the educator's information literacy improved in order to ensure that he acquired the ability of knowledge through the network in order to ensure the smooth progress of network teaching.

\section{Problems in the Development of Informational Orthopedics in China}

(1) As the regional economic, cultural, scientific and technological conditions, differences in the development of hospital information in the development of orthopedic orthodontic disorder is not synchronized, especially in western remote areas underdeveloped, weak information base, shortage of funds, lack of talent, digital inspection Treatment equipment configuration is lagging behind. In order to realize the orthodontic orthodonticization in the real sense, we should strengthen the knowledge and training of the leaders of the academic leaders, and take the scientific development concept as the guide, from the department construction to the technical construction to enhance the international advanced concept, the innovation concept is extremely important; (2) National and provincial (military) key construction specialist, including the top three hospitals, including the majority of hospital orthopedic information room to become the form of books and periodicals rarely, senior professionals to master a single foreign language, to be familiar with $6 \mathrm{~S}$ information technology and often in the online and books On the access to foreign scientific and technological information, the exchange of international information on the podium is not much, the correct master of foreign language writing ability less, according to "Chinese Journal of orthopedics" statistics of the annual acceptance of the article about $20 \%-30 \%$ of the English ${ }^{[6]}$. The translation of Chinese content is incorrect, grammatical errors, spelling errors and medical term selection errors and so on need to be modified or can not be used. In addition, there is little time to pay attention to the development of $6 \mathrm{~S}$ information technology and the development of communication and communication technology. Therefore, it is not timely and inadequate to master the domestic specialist information, and to a certain extent, The Weak Links in the Modernization of Departments. But also to see, in recent years with China's medical and health system reform continues to deepen, Quality medical service information resources gradually improved. According to the 2010 Asia-Pacific Medical and Health Information Technology Expo information, more than 90\% of China's county and above the county medical institutions to establish electronic medical records, according to the Chinese Hospital .Association Information Committee survey shows that the application of tertiary hospitals in the test fee system, Drug management system and the proportion of registered systems were $93 \%, 91 \%$ and $84 \%$, the application of hospital workstation system and the proportion of registered systems were $80 \%$ and $63 \%$, more than half of medical institutions in the hospital information construction investment last year 1 million Yuan. These data will undoubtedly speed up the development of China's information orthopedic orthodontic development to create new opportunities.

\section{Discussion}

Hospital information management system is China in recent years with the computer gradually spread and widely used in the clinical management system, the use of management system, reducing the workload of health care workers, shortening the patient waiting time. And the management system is a computer as a criterion to reduce the human error, although the patient's condition on the mastery remains to be improved, indicating that the information management system in the clinical play a great role. Effectively improve the efficiency of outpatient registration, eliminating the waiting time for patients waiting in line. In the infusion chamber through the process optimization, can effectively reduce the patient pay and bring back and forth with drugs. Improve the quality of medical records management, reducing the medical error. Through the information management system, reducing the medical staff due to the increase in the number of patients caused by errors, and reduces the waste of hospitalized patients. With the increasing 
demand for medical services, the demand for treatment of medical services has been gradually improved. The information management system has become the necessary path of social development and the development direction of hospital information management. From the current information management situation in China, based on the integrated information system, through the integration of medical resources information, the establishment of a strong clinical support system to high-quality, efficient, low-cost medical service process, as the center of the quality of medical services truly realize the standardization of all medical information, information technology. This is for the patients, health care workers and hospitals are a great role.

At present, we must realize that the information management system is heavily dependent on the computer system, the system once the external attack, it will cause the whole work paralyzed, in order to ensure data security, we have to backup the hard disk, tape backup and other measures, and to Regularly integrate, upgrade, and maintain information.

\section{References}

[1] Price CC, Levy SA, Tanner J, et al. Orthopedic Surgery and Post-Operative Cognitive Decline in Idiopathic Parkinson's Disease: Considerations from a Pilot Study. J Parkinsons Dis. 2015. 5(4): 893-905.

[2] Lee KH, Kim JY, Kim JW, Park JS, Lee KW, Jeon SY. Influence of Ketamine on Early Postoperative Cognitive Function After Orthopedic Surgery in Elderly Patients. Anesth Pain Med. 2015. 5(5): e28844.

[3] Beaulé PE, Frombach AA, Ryu JJ. Working toward benchmarks in orthopedic OR efficiency for joint replacement surgery in an academic centre. Can J Surg. 2015. 58(6): 408-13.

[4] Goradia V, Cannon WD. Computer navigation and robotics in orthopedic surgery. Sports Med Arthrosc. 2014. 22(4): 201.

[5] Morgan M, Aydin A, Salih A, Robati S, Ahmed K. Current Status of Simulation-based Training Tools in Orthopedic Surgery: A Systematic Review. J Surg Educ. 2017. 74(4): 698-716.

[6] Gibbs DB. Surgical Simulation in Orthopedic Surgery Residency. Am J Orthop (Belle Mead NJ). 2016. 45(7): E426-E428. 